\title{
LOS SUELOS DEL PAISAJE ALTO-ANDINO DE SANTA ISABEL (TOLIMA, COLOMBIA) ¿SON SUMIDEROS DE CARBONO ORGANICO?
}

\section{SOILS IN HIGH-LAND LANDSCAPES OF SANTA ISABEL (TOLIMA, COLOMBIA), ARE THEY A CARBON SINK?}

\author{
Andrés Sebastián Rojas ${ }^{1}$, Hernán J. Andrade ${ }^{2,4}$, Milena A. Segura M. ${ }^{3}$
}

${ }^{1}$ Ingeniero Agrónomo, Grupo de Investigación PROECUT, Facultad de Ingeniería Agronómica. Universidad del Tolima, e-mail: asrojas@ut.edu.co; ${ }^{2}$ Ingeniero Agrónomo, MSc, PhD, Grupo de Investigación PROECUT, Facultad de Ingeniería Agronómica. Universidad del Tolima; ${ }^{3}$ Ingeniera Forestal, M.Sc, Grupo de Investigación PROECUT, Facultad de Ingeniería Forestal. Universidad del Tolima, e-mail: masegura@ut.edu.co; ${ }^{4}$ Autor para correspondencia. Universidad del Tolima, Barrio Santa Helena, Ibagué, Tolima, e-mail: hjandrade@ut.edu.co

\author{
Rev. U.D.C.A Act \& Div. Cient. 21(1): 51-59, Enero-Junio, 2018 \\ https://doi.org/10.31910/rudca.v21.n1.2018.662
}

\section{RESUMEN}

El cambio climático global es uno de los problemas más graves de la humanidad, causado, principalmente, por el aumento de $\mathrm{CO}_{2}$ y otros gases de efecto invernadero en la atmósfera. Se reconoce que las emisiones de estos gases pueden ser reducidas disminuyendo su emisión o aumentando su remoción y depósito, en sumideros terrestres. El suelo es el mayor sumidero de carbono (C) y mitiga su creciente concentración atmosférica. Los paisajes rurales alto-andinos retienen importantes cantidades de $\mathrm{C}$ orgánico del suelo (COS), teniendo un papel fundamental en su ciclo global, pero ha sido poco valorado. El objetivo de este estudio fue estimar el COS en los principales usos de la tierra y los impactos del cambio de uso en paisajes alto-andinos en Santa Isabel - Tolima, Colombia. Se evaluaron tres usos del suelo: (i) cultivos agrícolas (CA), (ii) sistemas silvo-pastoriles de pasturas con árboles dispersos (SSP) y (iii) bosques nativos (BN), estimando la densidad aparente, por el método del cilindro y la concentración de COS, a una profundidad de $0-40 \mathrm{~cm}$. La densidad aparente, se incrementó notablemente, al cambiar de BN a CA y SSP $\left(0,35\right.$ a 0,61 y $0,65 \mathrm{~g} / \mathrm{cm}^{3}$, respectivamente). Contrastando, la concentración de COS, se redujo al convertir BN a usos agropecuarios (8,9 a 5,8\%). Los BN almacenaron más C que los CA y SSP $(122,4 ; 79,6$ y $79,8 \mathrm{t} /$ ha, respectivamente), causando una emisión potencial de 157 t CO$/$ ha, en caso de deforestación. Procesos contrarios implican captura de $\mathrm{C}$ atmosférico. La conservación de $\mathrm{BN}$ alto-andinos debe estar altamente considerados en las políticas nacionales y regionales.

Palabras clave: Agroforestería, bosques tropicales, cultivos (agricultura), densidad del suelo.

\section{SUMMARY}

Global climate change is one of the most serious problems of mankind, caused mainly by the increase of $\mathrm{CO}_{2}$ and other greenhouse gases in the atmosphere. It is recognized that net emissions of these gases can be reduced either by reducing their emission or by increasing their removal by depositing in terrestrial sinks. The soil is the largest carbon (C) sink and mitigates its increase in atmospheric concentration. The rural landscapes in high-land andean zones retain significant amounts of organic soil C (SOC), playing a key role in its global cycle. The objective of this study was to estimate the SOC stocks in the main land uses, and the impacts of land use change in high-land andean landscapes in Santa Isabel, Tolima, Colombia. Three land uses were evaluated: (i) agricultural crops (AC), (ii) silvopastoral systems of pastures with dispersed trees (SPS), and (iii) native forests (NF), estimating bulk density (BD) by cylinder method and the concentration of SOC to a depth of $0-40 \mathrm{~cm}$. The BD notably increased by changing from NF to AC and SPS (0.35 to 0.61 and $0.65 \mathrm{~g}$ / $\mathrm{cm}^{3}$, respectively). Contrasting, the concentration of SOC was reduced when NF change to agricultural and livestock uses (8.9 to 5.8\%). The NF stocked more $\mathrm{C}$ than AC and SPS (122.4, 79.6 and 79.8t/ha, respectively), causing a potential emission of $157 \mathrm{t} \mathrm{CO}_{2} / \mathrm{ha}$ in case of deforestation. Opposite processes imply capture of atmospheric carbon. The conservation of NF in high-land Andean zones must be highly considered in national and regional policies.

Key words: Agroforestry, tropical forests, crops (agriculture), soil density. 


\section{INTRODUCCIÓN}

El cambio climático global, uno de los problemas más graves de la humanidad, se atribuye, principalmente, al incremento de la concentración de gases de efecto invernadero (GEI) en la atmósfera, tal como el dióxido de carbono $\left(\mathrm{CO}_{2}\right)$, el metano $\left(\mathrm{CH}_{4}\right)$ y el óxido nitroso $\left(\mathrm{N}_{2} \mathrm{O}\right)$ (IPCC, 2003). Según el Departamento Nacional de Planeación y el Banco Interamericano de Desarrollo (NDP-BID, 2014), el país se ve constantemente afectado por eventos hidro-meteorológicos extremos, en gran parte de su territorio: las inundaciones, los deslizamientos, las granizadas y las olas de calor han incrementado su frecuencia en los últimos años, afectando las actividades económicas y a las personas de las zonas más sensibles. Las proyecciones de escenarios futuros estiman un incremento en la temperatura de alrededor de $4^{\circ} \mathrm{C}$, en algunas zonas del país, lo cual, podría incidir negativamente en la salud, al hacer más comunes a enfermedades, como el dengue y la malaria, máxime, en las zonas más cálidas (Gutiérrez \& Espinosa, 2010). Las regiones tropicales alto-andinas han sido identificadas como vulnerables al cambio climático, por un mayor calentamiento en zonas de alta elevación, en esta parte del mundo (Büchler et al. 2004).

El suelo es el mayor sumidero de carbono (C) y posee el potencial de ser incrementado y, de esta manera, mitigar el cambio climático (FAO, 2002). Por esta razón, muchos de los proyectos de captura de $\mathrm{C}$ incluyen este componente, tal como aquellos de aforestación y de reforestación, empleando bosques y sistemas agroforestales (Pearson et al. 2005). De otro lado, los ecosistemas alto-andinos, debido a la génesis de sus suelos y al clima reinante, presentan un alto contenido de COS, al tener bajas tasas de mineralización; sin embargo, estas grandes cantidades de C están siendo amenazadas por cambios de uso del suelo, que implican la pérdida de la cobertura boscosa hacia sistemas de producción sin árboles, tal como pasturas y cultivos de papa (Otero et al. 2011; Andrade et al. 2014). La dinámica del COS en paisajes alto-andinos ha sido poco estudiada, principalmente en Colombia, aunque Andrade et al. (2014) afirman que las pasturas extensivas pueden promover su acumulación, en sitios de altura.

Los pocos estudios hacen desconocer el impacto del uso del suelo en la dinámica del COS, lo que tiene implicaciones para el ciclo global del $\mathrm{CO}_{2}$. Este estudio tuvo como objetivo estimar el impacto de la copa y uso del suelo en el almacenamiento de COS y el impacto de potenciales cambios de uso del suelo, tal como la deforestación, en la fijación de carbono o emisión de GEI.

\section{MATERIALES Y MÉTODOS}

Área de estudio: El estudio, se realizó en nueve fincas de productores, ubicadas en las veredas la Yuca y Buena Vis- ta, del Municipio de Santa Isabel, localizado en el Tolima, Colombia (Figura 1). El municipio, se encuentra entre los $4^{\circ} 39^{\prime} 20^{\prime \prime}-4^{\circ} 48^{\prime} 38^{\prime \prime}$ N $\mathrm{N}$ y los $75^{\circ} 22^{\prime} 54^{\prime \prime}-75^{\circ} 0^{\prime} 48^{\prime} \mathrm{O}$, a una altitud de $2.400 \mathrm{~m}$ y se sitúa en un área de Bosque Húmedo Montano Bajo (bh-mb), según Holdridge (1996), dominada por ganadería y por agricultura. La zona de estudio presenta una temperatura promedio de $16^{\circ} \mathrm{C}$, con precipitación media anual de 1.800mm (IDEAM, 2017). El relieve es muy quebrado a escarpado (75-100\% de pendiente) y presenta suelos bien drenados, profundos y con alta susceptibilidad a la erosión. Los suelos de este estudio son derivados de cenizas volcánicas, los cuales, poseen características físico-químicas especiales, mediana fertilidad, buena estabilidad estructural, ph ácidos, fijadores de fósforo y de baja mineralización relativa de nitrógeno (Fajardo, 2005) y hacen parte de la unidad MKB, consociación Alic Hapludans (IGAC, 2004).

Diseño experimental: Se empleó un diseño experimental completamente al azar, evaluando tres de los usos del suelo más dominantes: 1) cultivo agrícola (CA) (tomate de árbol Solanum betacea Cav.); 2) sistemas silvopastoriles de pasturas de kikuyo (Pennisetum clandestinum), con árboles nativos de Weinmannia balbisiana Kunth, Eugenia spp., Styrax spp. y Solanum spp. dispersos (SSP) y 3) bosques nativos (BN), con cinco repeticiones por uso. En los SSP, también se estudió el efecto de la posición respecto a la copa arbórea en el COS, tomando tres posiciones: bajo, borde y fuera de la copa. El COS, se estimó a una profundidad de $0-40 \mathrm{~cm}$, siguiendo la Guía de Buenas Prácticas del IPCC (2003) y las recomendaciones de Andrade \& Segura (2017). La densidad aparente (DA), se estimó tomando tres muestras por repetición por el método del cilindro (IPCC, 2003; Andrade \& Segura, 2017) y secándolas hasta peso constante $\left(105^{\circ} \mathrm{C}\right)$. La concentración de COS, se estimó de una muestra compuesta de 10 sub-muestras por repetición, con un barreno helicoidal, analizada por el método de Walkley \& Black (1934). No se consideró la proporción de fragmentos gruesos, por considerarse despreciable $(<5 \%)$.

Impacto de potenciales cambios de uso en existencias de COS: Las alteraciones del COS, por efecto de posibles cambios de uso del suelo, se estimaron calculando el almacenamiento de esta variable en función de una masa de suelo en lugar de una profundidad fija (Mannetje et al. 2008), tomando la DA de los BN, como línea de base o situación de referencia. Se construyó una matriz simulando cambios de uso entre los tres sistemas, calculado como la diferencia en el almacenamiento de COS, entre el uso actual y el futuro. Estas diferencias, se multiplicaron por 3,67, para estimar los cambios en términos de $\mathrm{CO}_{2}$ e (IPCC, 2003), en donde los valores positivos implican captura adicional de $\mathrm{C}$, mientras que los negativos reflejan una emisión (Andrade et al. 2016). 


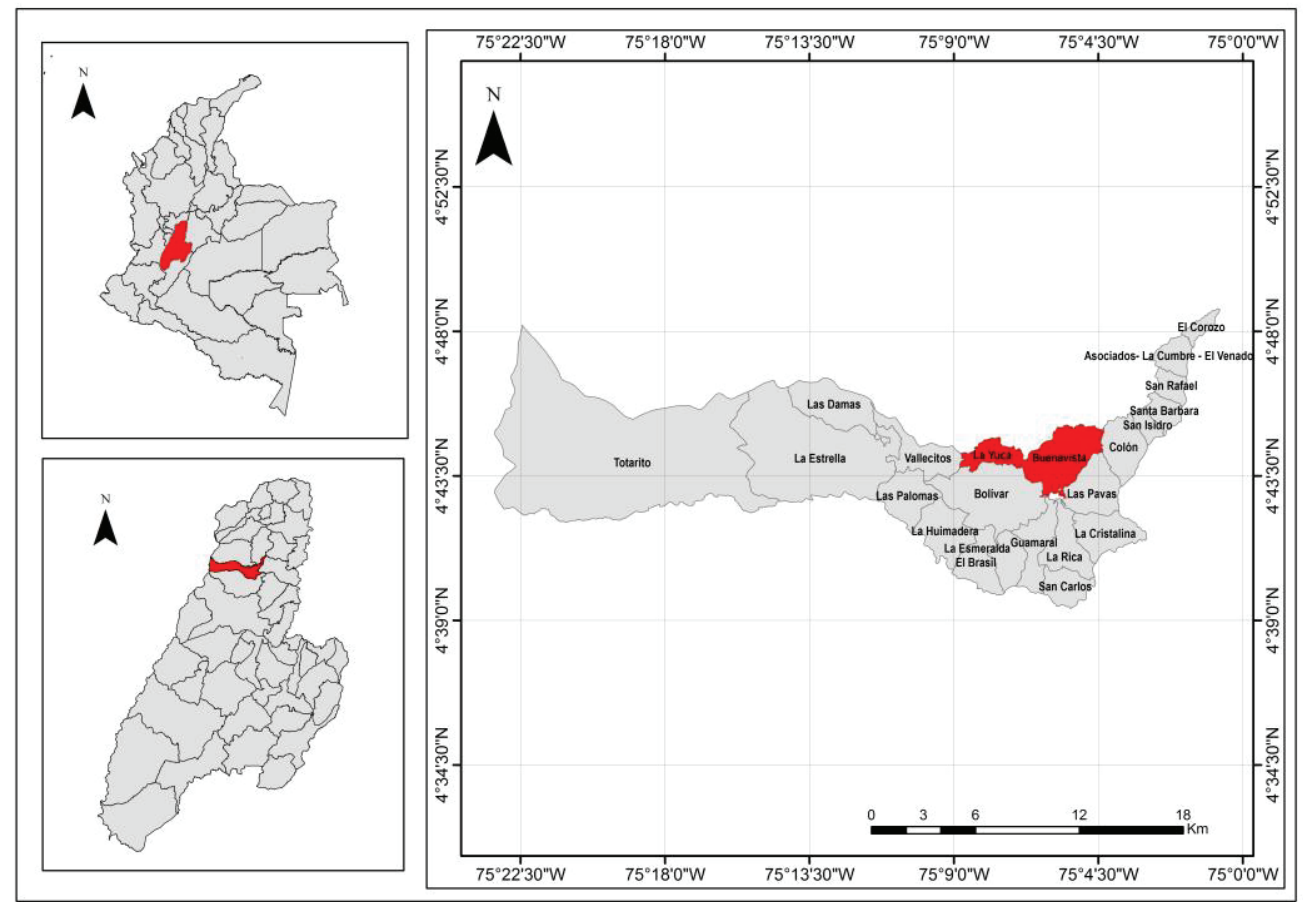

Figura 1. Localización del área de estudio en el Municipio de Santa Isabel, Tolima, Colombia.

Análisis estadístico: Se comprobaron los supuestos del diseño experimental y se realizaron pruebas de comparación de medias de Fisher a los tratamientos simples (sistemas de uso de la tierra y posición respecto a la copa) y factoriales (combinando estos tratamientos simples), con una confianza del 95\%, cuando se detectaron diferencias en el análisis de varianza. Adicionalmente, se realizó un análisis de correlación y de regresión lineal, para establecer la relación entre la DA y la concentración de COS, en los diferentes usos del suelo. Los análisis estadísticos, se realizaron con InfoStat (Di Rienzo et al. 2011).

\section{RESULTADOS Y DISCUSIÓN}

Efecto de la copa arbórea en el COS: En los SSP, no se detectaron diferencias estadísticas en la concentración de COS entre posiciones respecto a la copa: entre 5,4 y $6,0 \%$ bajo y fuera de la copa, respectivamente (Figura 2). De la misma forma, tampoco se observaron diferencias significativas en las existencias de COS en las diferentes posiciones: 139,0 a 153,0t/ha (Figura 2), pero el COS bajo la copa es inferior al borde y fuera de ésta. En este caso, la copa de los árboles no está afectando la acumulación de COS en pasturas. Esto se podría deber a que, bajo los árboles, debido a la sombra, probablemente, habrá menor biomasa de la pastura y de raíces finas (diámetro $<2 \mathrm{~mm}$ ), que puedan contribuir al COS al momento de senescer (Jobbagy \& Jackson, 2000; Andrade et al. 2008; Andrade et al. 2014).
De otro lado, la baja disponibilidad de forraje bajo la copa de los árboles, causado por una mayor sombra (Andrade et al. 2008), ocasionaría que los animales permanezcan menos tiempo bajo la copa, reduciendo el aporte de raíces muertas y hojarasca (Pezo \& Ibrahim, 1998). Esta interacción compensaría otros beneficios de las copas, como la menor temperatura de la superficie del suelo.

Densidad aparente (DA): La DA fue significativamente mayor en los suelos de SSP y CAque en los BN $(0,65$ vs 0,61 vs $0,35 \mathrm{~g} / \mathrm{cm}^{3}$, respectivamente) (Figura 3), es decir, al cambiar de BN a SSP o CA, se incrementa la DA del suelo, en un $80 \%$, lo cual, tiene grandes impactos para la salud de los suelos, en términos de fertilidad y de propiedades físicas de los suelos y las existencias de COS. En teoría, la DA en suelos con propiedades ándicas es menor a $0,90 \mathrm{~g} / \mathrm{cm}^{3}$ (Chinchilla et al. 2011), caso contrario se observa en suelos de la Cordillera de Talamanca de Costa Rica, donde esta variable osciló entre 0,6 y $1,3 \mathrm{~g} / \mathrm{cm}^{3}$, lo que denota la poca influencia de la ceniza volcánica o discontinuidad litológica en los horizontes sub-superficiales (Chinchilla et al. 2011).

Otros estudios han reportado valores contrastantes en la DA; por ejemplo, Fajardo (2005) encontró $0,70 \mathrm{~g} / \mathrm{cm}^{3}$ en suelos derivados de cenizas volcánicas de la zona cafetera de Colombia, lo que coincide con estos hallazgos. En este estudio, se encontró que la DA de los BN fue $0,35 \mathrm{~g} / \mathrm{cm}^{3}$, similar a los reportes de Zúñiga et al. (2013), en bosques no intervenidos 


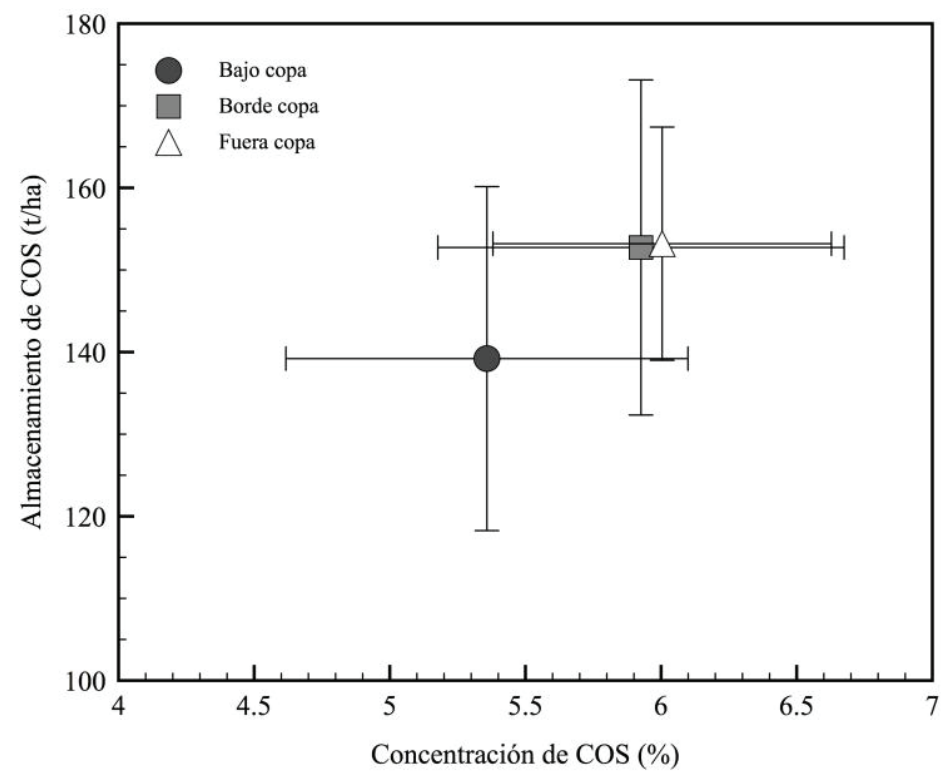

Figura 2. Efecto de copa arbórea en el almacenamiento y en la concentración de carbono orgánico del suelo (COS), a una profundidad de $0-40 \mathrm{~cm}$, en sistemas silvopastoriles de pasturas con árboles dispersos en potreros, en paisajes alto-andinos en Santa Isabel, Tolima, Colombia. Las barras de error corresponden a error estándar.

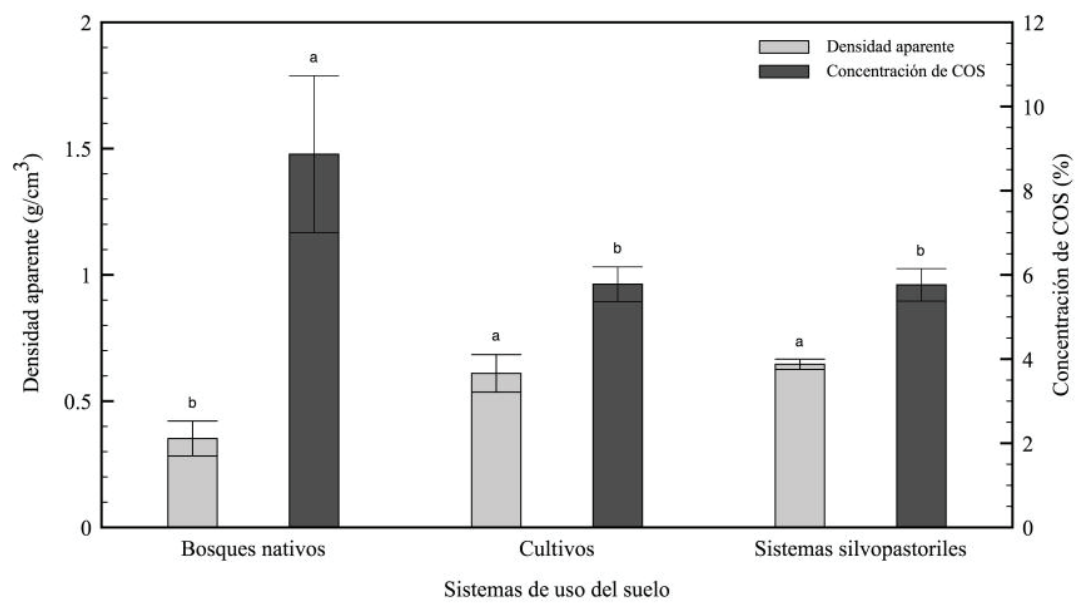

Figura 3. Densidad aparente y concentración de carbono orgánico del suelo (COS), en sistemas de uso del suelo en paisajes alto-andinos de Santa Isabel, Tolima, Colombia. Las barras de error corresponden a error estándar. Letras diferentes indican diferencias estadísticas $(\mathrm{p}<0,05)$.

en el Parque Nacional Chingaza y el Parque Nacional de los Nevados: 0,2 y $0,6 \mathrm{~g} / \mathrm{cm}^{3}$, respectivamente. De otra parte, Andrade et al. (2014) reportaron una DA promedio de 0,63g/ $\mathrm{cm}^{3}$, en pasturas activas el páramo de Anaime, Tolima, lo cual, resulta muy similar con estos estimados en SSP. La DA de cultivos agrícolas $\left(0,61 \mathrm{~g} / \mathrm{cm}^{3}\right)$ concuerda con Carvajal et al. (2009), Paz \& Sánchez (2007) y Alvarado et al. (2013), en suelos derivados de cenizas volcánicas $(0,67 ; 0,69$ y $0,88 \mathrm{~g} /$ $\mathrm{cm}^{3}$, respectivamente).

El aumento de DA en SSP y CA es, probablemente, debido al sobrepastoreo y la ausencia de cobertura vegetal sobre el suelo, ya que los hace más expuestos a fenómenos que alteran su composición química, física y biológica, lo que 
los hace propensos a la compactación (FAO, 2002; Fajardo, 2005). Neill et al. (1997) encontraron también incrementos de la DA en pasturas, luego de la deforestación en la Amazonia brasileña; por otro lado, diversos estudios han demostrado que un incremento en la diversidad y en la abundancia de plantas reduce la DA (Becknell \& Powers, 2014; Andrade et al. 2016), lo cual, mejora sustancialmente sus propiedades físicas, como la porosidad, la infiltración y la conductividad hidráulica. Shukla et al. (2006) afirman que la aireación y la agregación, variables que se relacionan estrechamente con la DA y la concentración de COS, son los factores más determinantes de la calidad de un suelo.

Concentración de carbono orgánico del suelo (COS): La concentración de COS fue estadísticamente superior en los BN, superando a SSP y CA ( 8,9 vs 5,8 vs $5,8 \%$, respectivamente) (Figura 3). En otras palabras, la deforestación reduciría la concentración de COS en un $35 \%$, en promedio. El rango de esta variable fue de 3,7 a $15,7 \%$, lo cual, coincide con los amplios reportes de Chinchilla et al. (2011), en suelos derivados de cenizas volcánicas, en la Cordillera de Talamanca, de Costa Rica (0,1 - 14,1\%). Entretanto, Fajardo (2005) reportó equivalentes de COS, de 7,0\%, en suelos derivados de cenizas volcánicas, de la zona cafetera de Colombia.

Los resultados de concentración de COS de otros autores son altamente contrastantes. Por un lado, en bosques no intervenidos en los Parques Nacionales de los Nevados y de Chingaza, se halló 1,8 y 6,9\% de concentración de COS, respectivamente (Zúñiga et al. 2013), que son ligeramente inferiores a estos resultados, en BN (8,9\%). En pasturas, Andrade et al. (2014) reportaron 4,6\%, en el páramo de Anaime, Tolima, lo que también resultó levemente menor a esta investigación (5,8\%), mientras que Farley et al. (2004) esti- maron en 4,2\% la concentración de COS, entre 0 y $10 \mathrm{~cm}$, en los Andes ecuatorianos. Los CA presentaron 5,8\% en esta variable, mientras que Alvarado et al. (2013) reportaron valores muy inferiores en cafetales, pero Paz \& Sánchez (2007) y Carvajal et al. (2009) estimaron valores muy cercanos (2,1; 5,6 y $6,4 \%$, respectivamente). En contraste, en el páramo de Sumapaz, Colombia, se estimó $11,1 \%$ de COS en los primeros $25 \mathrm{~cm}$ de suelo en cultivos de papa (Montes-Pulido et al. 2017), lo cual, resulta extremadamente superior a lo encontrado en estos CA; sin embargo, se debe aclarar que en páramos hay menores tasas de descomposición de la materia orgánica (Andrade et al. 2014).

Las bajas concentraciones de COS en los suelos agropecuarios (SSP y CA), se podrían atribuir a la constante remoción del mantillo superficial por las labores culturales y la erosión causada por las altas pendientes; sin embargo, se ha observado que el COS se puede incrementar con la edad de la pastura y un adecuado manejo del pastoreo (Neill et al. 1997; Andrade et al. 2014). Caso contrario sucede en los $\mathrm{BN}$, pues la erosión es menor y la alta producción de necromasa y las bajas temperaturas promueven la acumulación de COS (Lizcano et al. 2006; Andrade et al. 2014).

No se encontró una estrecha relación entre la concentración de COS y la DA en los sistemas evaluados $(r=0,59)$ (Figura 4); sin embargo, aunque estadísticamente no es significativo, se observa una leve tendencia en la reducción de la DA, a medida que se incrementa la concentración de COS, tal como encontraron Alvarado et al. (2013) y Andrade et al. (2014; 2016). Este comportamiento representa un beneficio productivo y ambiental para estos paisajes, con los usos del suelo estudiados.

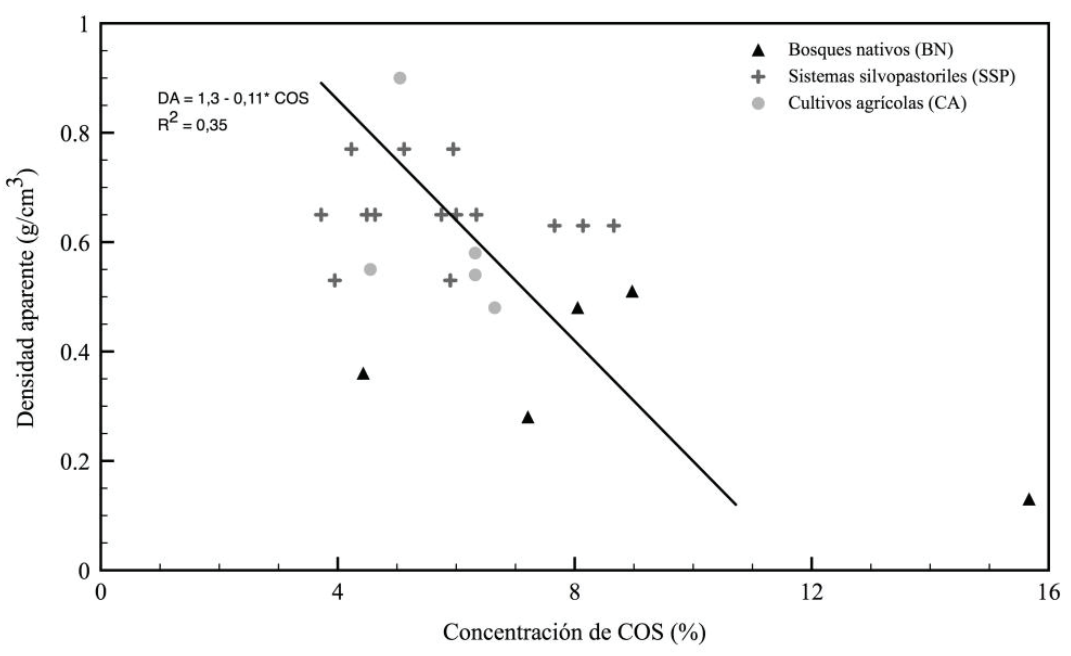

Figura 4. Relación entre la concentración de carbono orgánico (COS) y la densidad aparente a una profundidad de 0-40cm del suelo, en sistemas de uso del suelo en paisajes alto-andinos de Santa Isabel, Tolima, Colombia. 
Existencias de COS: No se detectaron diferencias estadísticas en las existencias de COS entre los sistemas evaluados; no obstante, en promedio los SSP almacenaron más $C$ que los CA y BN (148,2 vs 138,2 vs 112,8 t/ha, respectivamente) (Figura 5). Es de aclarar, que la DA empleada fue la propia de cada suelo, lo cual, implica que, procesos de compactación, incrementarían el volumen del suelo, al considerar una capa mayor de suelo, cuando se estima con una misma profundidad.
Los suelos en estos paisajes alto-andinos, bajo estos usos del suelo, almacenaron entre 63,3 a 217,1t/ha, en los primeros $40 \mathrm{~cm}$, los que coinciden con Chinchilla et al. (2011), en suelos derivados de cenizas volcánicas, en Talamanca, Costa Rica (6,5 - 262,8t/ha) y FAO (2002), en Andosoles $(112,8 \mathrm{t} / \mathrm{ha})$, pero difiere a los $243,6 \mathrm{t} / \mathrm{ha}$, en suelos derivados de cenizas volcánicas, de la zona cafetera de Colombia (Fajardo, 2005). En bosques no intervenidos en los Parques Nacionales de los Nevados y de Chingaza, se encontró 107,7

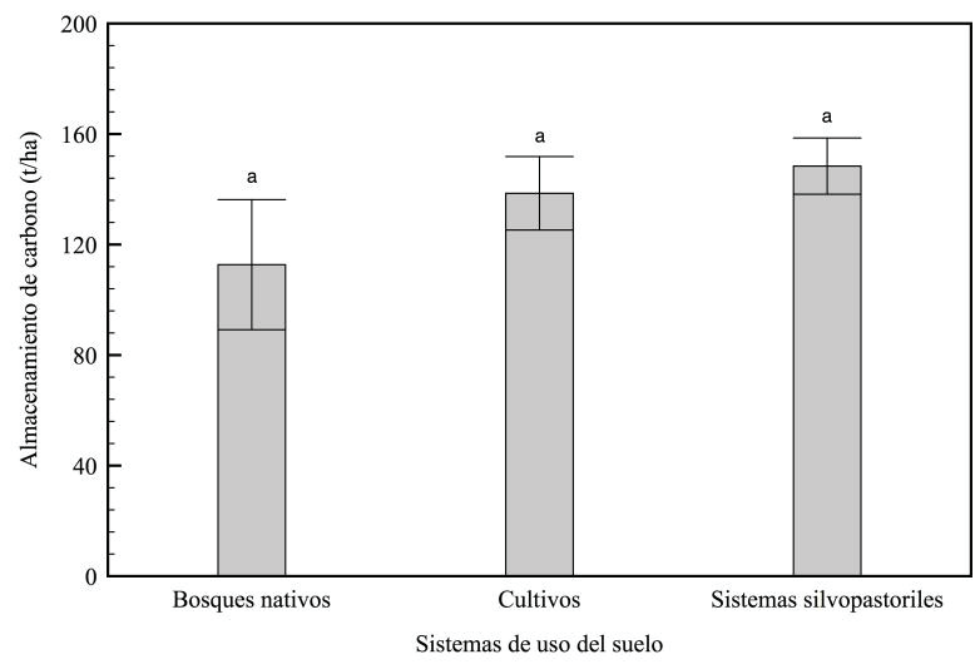

Figura 5. Existencias de carbono orgánico del suelo a una profundidad de $0-40 \mathrm{~cm}$, en bosques nativos, cultivos agrícolas y sistemas silvo-pastoriles, en paisajes alto-andinos de Santa Isabel, Tolima, Colombia. Las barras de error corresponden a error estándar. Letras iguales indican que no hay diferencias estadísticas $(p>0,05)$ entre sistemas.

y 137,5t/ha de COS, respectivamente (Zúñiga et al. 2013), siendo congruentes con estos hallazgos. En contraste, Andrade et al. (2014) reportaron 85,9t/ha, en pasturas del páramo de Anaime, Tolima, menor a los estimados para los SSP de Santa Isabel. Carvajal et al. (2009), Alvarado et al. (2013) y Montes-Pulido et al. (2017) estimaron existencias de COS similares en CA.

Alteraciones en las existencias de COS por cambios de uso del suelo: Al estimar las existencias de COS, con base en una misma masa, el COS en BN fue significativamente superior a SSP y CA $(122,4$ vs 79,6 vs 79,8 t/ha, respectivamente), es decir, al cambiar de SSP y de CA a BN, se estaría capturando una gran cantidad de $\mathrm{CO}_{2}$ de la atmósfera (Tabla 1) y se liberaría la misma cantidad, si se dieran procesos de deforestación. En este sentido, la aforestación o reforestación podrían causar la remoción, en el largo plazo, de cantidades similares de $\mathrm{CO}_{2}$ (IPCC, 2003). Estos resultados, aunque parecen lógicos, difieren de los hallazgos de Andrade et al. (2014), quienes reportaron una tendencia opuesta, ya que el paso de BN a pasturas activas con manejo extensivo, tendió a aumentar el COS, en cerca de 12,8t/ha, en el páramo de Anaime, Tolima, Colombia.

Zúñiga et al. (2013) reportaron pérdidas de 55,6t/ha de COS, por cambio de bosques primarios a bosques intervenidos, en el Parque Nacional Chingaza, mientras que encontraron ganancias de 16,7t/ha de COS, al cambiar bosques primarios a bosques intervenidos, en el Parque Nacional los Nevados. Desjardins et al. (1994) demostraron, en la Amazonia oriental, que el paso de BN a pasturas por 10 años causa pérdidas de 2,0t/ha de COS; mientras que Veldkamp (1994) calculó en 14,9t/ha las pérdidas por deforestación en Costa Rica. Los resultados de este estudio coinciden parcialmente con el metanálisis de Guo \& Gifford (2002), quienes encontraron reducciones del 42 y $59 \%$ cuando $\mathrm{BN}$ se convierten a pasturas y CA, respectivamente; mientras que cambios de $\mathrm{BN}$ y CA a pasturas incrementan el COS en un 8 y $19 \%$, respectivamente. El manejo también influye en el COS, ya que West \& Post (2002) afirman que el cambio de labranza convencional a no labranza, promueve la captura de 5,7t/ha/año. 
Tabla 1. Impacto del cambio de uso del suelo en el almacenamiento de carbono orgánico del suelo ( $\mathrm{C} \mathrm{CO}_{2} / \mathrm{ha}$ ), a una profundidad de 0 a $40 \mathrm{~cm}$, en paisajes alto-andinos en Santa Isabel, Tolima, Colombia.

\begin{tabular}{|c|c|c|c|c|c|}
\hline & & & \multicolumn{3}{|c|}{ Uso futuro } \\
\hline & & & $\begin{array}{l}\text { Bosques nativos } \\
\text { (BN) }\end{array}$ & $\begin{array}{l}\text { Cultivos agrícolas } \\
\text { (CA) }\end{array}$ & $\begin{array}{l}\text { Sistemas silvopastoriles } \\
\text { (SSP) }\end{array}$ \\
\hline & & & $122,4 \mathrm{t} \mathrm{C} / \mathrm{ha}$ & $79,8 \mathrm{t} \mathrm{C} / \mathrm{ha}$ & $79,6 \mathrm{t} \mathrm{C} / \mathrm{ha}$ \\
\hline \multirow{3}{*}{ 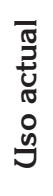 } & Bosques nativos (BN) & $122,4 \mathrm{t} \mathrm{C} / \mathrm{ha}$ & 0 & $-157,1$ & $-156,3$ \\
\hline & Cultivos agrícolas (CA) & $79,8 \mathrm{t}$ C/ha & 157,1 & 0 & 0,7 \\
\hline & Sistemas silvopastoriles (SSP) & $79,6 \mathrm{t} \mathrm{C} / \mathrm{ha}$ & 156,3 & $-0,7$ & 0 \\
\hline
\end{tabular}

Valores positivos corresponden a fijación de carbono en suelos, mientras que los valores negativos reflejan emisión de $\mathrm{CO}_{2}$ a la atmósfera.

Guo \& Gifford (2002) aseguran que, si un cambio de uso del suelo reduce el COS, el cambio inverso lo incrementa. Estos estimados de cambios en el COS demuestran la importancia ambiental de estos ecosistemas, ya que además se debe considerar el $\mathrm{C}$ en otros componentes, tal como biomasa y necromasa (Andrade et al. 2014) y otros servicios ambientales que ofrecen los $\mathrm{BN}$, principalmente, en paisajes alto-andinos, tal como la regulación hídrica, la conservación de la biodiversidad y del suelo (FAO, 2002).

Agradecimientos: Los autores agradecen al Comité Central de Investigaciones de la Universidad del Tolima. También, se agradece sinceramente a los productores de la zona que participaron en la investigación. Conflictos de intereses: El manuscrito fue preparado y revisado con la participación de todos los autores, quienes declaramos que no existe ningún conflicto de intereses que ponga en riesgo la validez de los resultados presentados. Financiación: Este estudio fue financiado por el Comité Central de Investigaciones de la Universidad del Tolima, mediante el proyecto código 530114.

\section{BIBLIOGRAFÍA}

1. ALVARADO, J.; ANDRADE, H.; SEGURA, M. 2013. Almacenamiento de carbono orgánico en suelos en sistemas de producción de café (Coffea arabica L.) en el municipio del Líbano, Tolima, Colombia. Colombia Forestal. (Colombia). 16(1):20-31.

2. ANDRADE, H.J.; BROOK, R.; IBRAHIM, M. 2008. Growth, production and carbon sequestration of silvopastoral systems with native timber species in the dry lowlands of Costa Rica. Plant and Soil. (Países Bajos). 308:11-22.
3. ANDRADE, H.; ESPINOSA, E.; MORENO, H. 2014. Impact of grazing in soil organic storage carbon in high lands of Anaime, Tolima, Colombia. Zootecnia Tropical. (Venezuela). 32(1):7-21.

4. ANDRADE, H.J.; SEGURA, M.A.; ROJAS, A.S. 2016. Carbono orgánico del suelo en bosques riparios, arrozales y pasturas en Piedras, Tolima, Colombia. Agronomía Mesoamericana. (Costa Rica). 27(2):1-9.

5. ANDRADE, H.J.; SEGURA, M.A. 2017. Colecta de datos de campo para inventarios de carbono. En: Estimación del carbono a partir de inventarios forestales nacionales: Buenas prácticas para la recolección, manejo y análisis de datos. Casanoves, F.; Cifuentes, M.; Chacón, M. (eds.). Serie técnica. Manual técnico No. 410. CATIE, Turrialba, Costa Rica. p.22-31

6. BECKNELL, J.M.; POWERS, J.S. 2014. Stand age and soils as drivers of plant functional traits and aboveground biomass in secondary tropical dry forest. Canadian J. Forest Research. (Canadá). 44(6):604-613.

7. BÜCHLER, B.; BRADLEY, R.; MESSERLI, B.; REASONER, M. 2004. Understanding climate change in mountains. Mountain Research and Development. (Estados Unidos).24(2):176-177.

8. CARVAJAL, A.F.; FEIJOO, A.; QUINTERO, H.; RONDÓN, M.A. 2009. Carbono orgánico del suelo en diferentes usos del terreno de paisajes andinos colombianos. Rev. ciencia del suelo y nutrición vegetal. (Chile). 9(3):222-235. 
9. CHINCHILLA, M.; MATA, R.; ALVARADO, A. 2011. Andisoles, inceptisoles y entisoles de la subcuenca del río Pirrís, región de los Santos, Talamanca, Costa Rica. Agronomía Costarricense. (Costa Rica). 35(1):83107.

10. DESJARDINS, T.; ANDREUX, F.; VOLKOFF, B.; CERRI, C.C. 1994. Organic carbon and ${ }^{13} \mathrm{C}$ contents in soils and soil size-fractions, and their changes due to deforestation and pasture installation in eastern Amazonia. Geoderma. (Países Bajos).61:103-118.

11. DI RIENZO, J.A.; CASANOVES, F.; BALZARINI, M.G.; GONZALEZ, L.; TABLADA, M.; ROBLEDO, C.W. 2011. Infostat, Grupo InfoStat, FCA, Universidad Nacional de Córdoba, Argentina. URL http://www. infostat. com. ar.

12. DNP-BID. 2014. Impactos económicos del cambio climático en Colombia - Síntesis. Bogotá, Colombia. 160p.

13. FAJARDO, N. 2005. El suelo. Uso y manejo de suelos. Ibagué, Colombia. Litoimagen impresores. 315p.

14. FAO. 2002. Captura de carbono en los suelos para un mejor manejo de la tierra. FAO, Roma, Italia. 61p.

15. FARLEY, K.A.; KELLY, E.F.; HOFSTEDE, R.G.M. 2004. Soil organic carbon and water retention after conversion of grasslands to pine plantations in the Ecuadorian Andes. Ecosystems. (Alemania). 7(7):729-739.

16. GUO, L.B.; GIFFORD, R.M. 2002. Soil carbon stocks and land use: a meta-analysis. Global Change Biology. (Reino Unido). 8(4):345-360.

17. GUTIÉRREZ, M.E.; ESPINOSA, T. 2010. Vulnerabilidad y adaptación al cambio climático. Diagnóstico inicial, avances, vacíos y potenciales líneas de acción en Mesoamérica. Banco Interamericano de Desarrollo, New York, USA, 81p.

18. HOLDRIDGE, L. 1996. Ecología basada en zonas de vida. $4^{a}$ reimpresión, San José, Costa Rica, Instituto Interamericano de Cooperación para la agricultura, 1996, c1978. Colección Libros y Materiales Educativos/IICA; no. 83. 216p.

19. IDEAM. 2017. Estaciones Meteorológicas: La Bodega y Santa Isabel. Sistema de Información Nacional Ambiental. Disponible desde Internet en: http://institucional.ideam.gov.co/jsp/loader.jsf?IServicio $=U$ - suarios $\&$ ITipo $=$ usuarios $\&$ IFuncion $=$ login $\& \quad($ con acceso el 16/02/2017).

20. INSTITUTO GEOGRÁFICO AGUSTÍN CODAZZI -IGAC-. 2004. Estudio General de Suelos y Zonificación de Tierras Departamento de Tolima. Bogotá DC.

21. INTERGOVERNMENTAL PANEL ON CLIMATE CHANGE -IPCC-. 2003. Good practice guidance for land use, land-use change and forestry. Disponible desde Internet en: http://www.ipcc-nggip-iges.or.jp 3 (con acceso el 16/02/2017).

22. JOBBAGY, E.G.; JACKSON, R.B. 2000. The vertical distribution of soil organic carbon and its relation to climate and vegetation. Ecological Applications. (Estados Unidos). 10(2):423-436.

23. LIZCANO, A.; HERRERA, M.; SANTAMARINA, J. 2006. Suelos derivados de cenizas volcánicas en Colombia. Revista Internacional de Desastres Naturales, Accidentes e Infraestructura Civil. (Puerto Rico). 6(2):167-198.

24. MANNETJE, L.; AMÉZQUITA, M.C.; BUURMAN, P.; IBRAHIM, M.A. 2008. Carbon Sequestration in Tropical Grassland Ecosystems. Wageningen, The Netherlands: Wageningen Academic Publishers, 221p.

25. MONTES-PULIDO, C.R.; RAMOS, J.J.; SAN JOSÉ, A.M. 2017. Estimation of soil organic carbon (SOC) at different soil depths and soil use in the Sumapaz paramo, Cundinamarca - Colombia. Acta Agronómica. (Colombia). 66(1):95-101.

26. NEILL, C.; MELILLO, J.M.; STEUDLER, P.A.; CERRI, C.C.; MORAES, J.F.L.; PICCOLO, M.C.; BRITO, M. 1997. Soil carbon and nitrogen stock following forest clearing for pasture in the southwestern Brasilian Amazon. Ecological Applications. (Estados Unidos). 7(4):1216-1225.

27. OTERO, J.D.; FIGUEROA, A.; MUÑOZ, F.A.; PEÑA, M.R. 2011. Loss of soil and nutrients by surface runoff in two agro-ecosystems within an Andean paramo area. Ecological Engineering. (Países Bajos). 37:2035-2043.

28. PAZ, E.; SÁNCHEZ, M. 2007. Relación entre dos sistemas de sombrío de café y algunas propiedades físicas del suelo en la meseta de Popayán. Acta Agronómica. (Colombia). 55(49):1-6. 
29. PEARSON, T.; WALKER, S.; BROWN, S. 2005. Sourcebook for land use, land-use change and forestry projects. Winrock International and the Biocarbon Fund of the World Bank. 57p.

30. PEZO, D.; IBRAHIM, M. 1998. Sistemas silvopastoriles. Módulo de Enseñanza Agroforestal No. 2. Proyecto Agroforestal CATIE/GTZ. Turrialba, Costa Rica. 275p.

31. SHUKLA, M.K.; LAL, R.; EBINGER, M. 2006. Determining soil quality indicators by factor analysis. Soil and Tillage Research. (Países Bajos). 87:194-204.

32. VELDKAMP, E. 1994. Organic carbon turnover in three tropical soils under pasture after deforestation. Soil Science Society of America J. (Estados Unidos). 58:175-180.
33. WALKLEY, A.; BLACK, C.A. 1934. An examination of the Degtajareff's method for determining soil organic matter and a proposed modification of the chromic acid titration method. Soil Science. (Estados Unidos). 37:29-38.

34. WEST, T.O.; POST, W.M. 2002. Soil organic carbon sequestration rates by tillage and crop rotation: a global data analysis. Soil Science Society of America J. (Estados Unidos). 66:1930-1946.

35. ZÚÑIIGA, O.; PEÑA, E.J.; TORRES, A.M.; CUERO, R.; PEÑA, J.A. 2013. Assessment of the impact of anthropic activities on carbon storage in soils of high montane ecosystems in Colombia. Agronomía Colombiana. (Colombia). 31(1):112-119.

Recibido: Febrero 2 de 2018

Aceptado: Mayo 25 de 2018

\section{Cómo citar:}

Rojas, A.S.; Andrade, H.J.; Segura M., M.A. 2018. ¿Son los suelos de paisajes alto-andinos de Santa Isabel (Tolima, Colombia) sumideros de carbono orgánico? Rev. U.D.C.A Act. \& Div. Cient. 21(1): 51-59. 